\title{
Relationship between EEG Alpha3/Alpha2 Ratio and the Nucleus Accumbens in Subjects with Mild Cognitive Impairment
}

\author{
Moretti DV *, Paternicò D, Binetti G, Zanetti O and Frisoni GB
}

IRCCS S. Giovanni di Dio Fatebenefratelli, Brescia, Italy

\begin{abstract}
Background: The nucleus accumbens (NAc) has been associated with impulsive behavior in subjects with early cognitive impairment; grey matter (GM) changes of basal ganglia have been demonstrated to be involved in Alzheimer's disease (AD). Moreover, the increase of EEG alpha3/alpha2 ratio has been associated with ADconverters subjects with mild cognitive impairment $(\mathrm{MCl})$.
\end{abstract}

Objective: To study the association of EEG marker with specific GM changes of NAc in subjects with MCl

Methods: 74 adult subjects with mild cognitive impairment underwent EEG recording and high resolution 3D magnetic resonance imaging (MRI). The alpha3/alpha2 ratio was computed for each subject. Three groups were obtained according to increasing tertiles values of alpha3/alpha2. Grey matter density differences between groups were investigated using a Voxel Based Morphometry technique.

Results: Subjects with lower a3/a2 and middle a3/a2 ratio ratio showed greater gray matter reduction in the NAc bilaterally when compared to subjects with higher a3/a2 ratio.

Conclusion: The a3/a2 ratio was associated with increase of grey matter density inside the $\mathrm{NAc}$ in $\mathrm{MCl}$ subjects at major risk to develop AD.

\section{Introduction}

EEG have been demonstrated a reliable diagnostic tool in dementia research $[1,2]$. The increase of high alpha relative to low alpha power has been recently demonstrated a reliable EEG marker of hippocampal atrophy as well as conversion of patients with mild cognitive impairment (MCI) in Alzheimer's disease (AD; [3]).

Impulsive behaviors are frequently described in brain-damaged patients, including patients with AD. More specifically, a significant increase in urgency, lack of premeditation, and lack of perseverance was noted, whereas a decrease in sensation seeking was observed in these patients [4]. Furthermore, this increase of impulsivity on urgency, lack of premeditation, and lack of perseverance was not associated with global cognitive impairment as assessed by the Mini Mental State Examination (MMSE; [5]) or the Mattis Dementia Rating scale [6], whereas lower sensation seeking was associated with a lower score on the MMSE [4,7].

The nucleus accumbens (NAc) is a key component of the neural processes regulating impulsivity [8]. Data from imaging studies [8] suggest the involvement of the NAc in impulsive choice. In particular an increase in the activity of NAc has been associated with decisions involving immediate outcome [9-11], with decisions including intertemporal differences [12], with choices of shorter delays [13] as well as with switching to risk-seeking choices [14].

In the present study the association of EEG indexes with grey matter (GM) changes in NAc has been studied in subjects with MCI. The working hypothesis was that modifications of alpha3/alpha2 power ratio could be underpinned by different deep brain structures. Results show that subjects with higher a3/a2 ratios when compared to subjects with lower and middle a3/a2 ratios showed minor atrophy in the NAc bilaterally. The results are discussed in the light of possible relationship with impulsive behavior in prodromal Alzheimer's disease patients. The subjects in this study are the same of a group analyzed in a previous paper (Moretti et al., 2012) [15]. The novelty of the present work was that the attention was more focused on the role of the NAc volumentric changes in subjects with mild cognitive impairment. This choice was made considering the growing consideration of recent literature about the function of the NAC as an important hub in behavioral regulation. In this view, the statistical threshold of the analysis was strenghtned (uncorrected threshold of $\mathrm{p}<0.001$ ).

\section{Materials and Methods}

\section{Subjects}

Seventy-four subjects with MCI were recruited from the memory Clinic of the Scientific Institute for Research and Care (IRCCS) of Alzheimer's and psychiatric diseases 'Fatebenefratelli' in Brescia, Italy. All experimental protocols had been approved by the local ethics committee. Informed consent was obtained from all participants or their caregivers, according to the Code of Ethics of the World Medical Association (Declaration of Helsinki).

\section{Diagnostic criteria}

Patients were selected from a prospective study on the natural history of cognitive impairment (the translational outpatient memory clinic-TOMC study) carried out in the outpatient facility of the National Institute for the Research and Care of Alzheimer's Disease

*Corresponding author: Vito Davide Moretti, IRCCS 'San Giovanni di Dio - FBF', Brescia; Italy, Tel: +39 0303501597; Fax: +39 0303533513; E-mail: davide.moretti@afar.it

Received February 08, 2013; Accepted March 30, 2013; Published April 09, 2013

Citation: Moretti DV, Paternicò D, Binetti G, Zanetti O, Frisoni GB (2013) Relationship between EEG Alpha3/Alpha2 Ratio and the Nucleus Accumbens in Subjects with Mild Cognitive Impairment. J Neurol Neurophysiol 4: 149 doi:10.4172/2155-9562.1000149

Copyright: (c) 2013 Moretti DV, et al. This is an open-access article distributed under the terms of the Creative Commons Attribution License, which permits unrestricted use, distribution, and reproduction in any medium, provided the original author and source are credited. 
(IRCCS Istituto Centro San Giovanni di Dio Fatebenefratelli, Brescia, Italy).

The project was aimed to study the natural history of non-demented persons with apparently primary cognitive deficits, i.e., deficits not due to psychic (anxiety, depression, etc.) or physical (hypothiroidism, vitamin $\mathrm{B} 12$ and folate deficiency, uncontrolled heart disease, uncontrolled conditions (diabetes, etc.) in the absence of functional impairment. The selection criteria has the aim to include as much as possible primary prodromal dementia due to neurodegenerative disorders. Demographic and cognitive features of the subjects in study are summarized in Table 1 . Selection criteria were extensively described elsewhere [15]. Briefly, patients were rated with a series of standardized diagnostic and severity instruments, including the MiniMental State Examination (MMSE; [5]), the Clinical Dementia Rating Scale (CDRS; [16]) the Hachinski Ischemic Scale (HIS; [17]) and the Instrumental and Basic Activities of Daily Living (IADL, BADL; [18]). In addition, patients underwent diagnostic neuroimaging procedures (magnetic resonance imaging, MRI), and laboratory testing to rule out other causes of cognitive impairment. All patients underwent: (i) semi-structured interview with the patient and - whenever possible with another informant (usually, the patient's spouse or a child of the patient) by a geriatrician or neurologist; (ii) physical and neurological examinations; (iii) performance-based tests of physical function, gait and balance; (iv) neuropsychological battery assessing memory (Babcock Story Recall - Rey-Osterrieth Complex Figure, Recall Auditory-Verbal Learning Test, immediate and delayed recall [19]) verbal and non-verbal memory, attention and executive functions (Trail Making Test B, A and B-A; Inverted Motor Learning-Clock Drawing Test; [19]), abstract reasoning thinking (Raven Colored Progressive Matrices; [19]), frontal functions (Inverted Motor Learning); language (Phonological and Semantic fluency-Token test [19]), and apraxia and visuo-constructional abilities (Rey-Osterrieth Complex Figure, Rey figure copy, Clock Drawing Test; [19]); (v) assessment of depressive symptoms by means of the Center for Epidemiologic Studies Depression Scale (CES-D; [20]). All the neuropsychological tests were standardized on Italian population, thus scores were compared to normative values with age, education and gender corrections in an Italian population. All subjects were right-handed. As the aim of our study was to evaluate the relationship between GM loss and alpha2/alpha3 ratios in MCI subjects, we did not consider the clinical subtype of MCI, i.e., Amnesic or non-amnesic, single or multiple domains.

\section{EEG recordings}

The EEG activity was recorded continuously from 19 sites by using electrodes set in an elastic cap (Electro-Cap International, Inc.) and positioned according to the 10-20 international systems (Fp1, Fp2, F7, F3, Fz, F4, F8, T3, C3, Cz, C4, T4, T5, P3, Pz, P4, T6, O1, and O2). In order to keep constant the level of vigilance, an operator controlled online the subject and the EEG traces, alerting the subject any time there were signs of behavioural and/or EEG drowsiness. The ground electrode was placed in front of Fz. The left and right mastoids served as reference for all electrodes. The recordings were used off-line to re-reference the scalp recordings to the common average. Re-referencing was done prior to the EEG artifact detection and analysis. Data were recorded with a band-pass filter of $0.3-70 \mathrm{~Hz}$, and digitized at a sampling rate of $250 \mathrm{~Hz}$ (BrainAmp, BrainProducts, Germany). Electrodes-skin impedance was set below $5 \mathrm{khz}$. Horizontal and vertical eye movements were detected by recording the electrooculogram (EOG). The recording lasted 5 min, with subjects with closed eyes. Longer recordings would have reduced the variability of the data, but they would also have increased the possibility of slowing of EEG oscillations due to reduced vigilance and arousal. EEG data were then analyzed and fragmented off-line in consecutive epochs of $2 \mathrm{~s}$, with a frequency resolution of $0.5 \mathrm{~Hz}$. The average number of epochs analyzed was 140, ranging from 130 to 150 . The EEG epochs with ocular, muscular and other types of artifact were preliminary identified by a computerized automatic procedure [21-29]. Two expert electroencephalographists manually double-checked and confirmed the automatic selections. The epochs with ocular, muscular and other types of artifacts were discarded.

\section{Analysis of individual frequency bands}

All recordings were obtained in the morning with subjects resting comfortably. Vigilance was continuously monitored in order to avoid drowsiness. A digital FFT-based power spectrum analysis (Welch technique, Hanning windowing function, no phase shift) computed ranging from 2 to $45 \mathrm{~Hz}$ - the power density of EEG rhythms with a $0.5 \mathrm{~Hz}$ frequency resolution. Two anchor frequencies were selected according to the literature guidelines [30,31], that is, the theta/alpha transition frequency (TF) and the individual alpha frequency (IAF) peak. These anchor frequencies were computed on the power spectra averaged across all recording electrodes. The TF marks the transition frequency between the theta and alpha bands, and represents an estimate of the frequency at which the theta and alpha spectra intersect. TF was computed as the minimum power in the alpha frequency range, since our EEG recordings were performed at rest. The IAF represents the frequency with the maximum power peak within the extended alpha range $(5-14 \mathrm{~Hz})$. Based on TF and IAF, we estimated the frequency band range for each subject, as follows: delta from TF-4 to TF- 2, theta from TF-2 to TF, low alpha band (alpha1 and alpha2) from TF to IAF, and high alpha band (or alpha3) from IAF to IAF +2 . The alpha1 and alpha2 bands were computed for each subject as follows: alpha1 from TF to the middle point of the TF-IAF range, and alpha2 from such middle point to the IAF peak [22-28]. The mean frequency range computed in MCI subjects considered as a whole are: delta 2.9$4.9 \mathrm{~Hz}$; theta $4.9-6.9 \mathrm{~Hz}$; alpha1 $6.9-8.9 \mathrm{~Hz}$; alpha2 $8.9-10.9 \mathrm{~Hz}$; alpha3 10.9-12.9 Hz; beta1 12.9-19.2 Hz; beta2 19.2-32.4; gamma 32.4-45. The relative power density for each frequency band was computed as the ratio between the absolute power and the mean power spectra from 2 to $45 \mathrm{~Hz}$. The relative band power at each band was defined as the mean of the relative band power for each frequency bin within

\begin{tabular}{|c|c|c|c|}
\hline & & & Alpha3/Alpha2 \\
\cline { 2 - 4 } & ALL & High & Middle \\
\hline Number of subjects & 74 & 18 & 38 \\
\hline Age, years & $69.4 \pm 7.6[52-85]$ & $70.4 \pm 6.7[60-85]$ & $68.4 \pm 8.2[52-83]$ \\
\hline Sex, female & $51(\%)$ & $13(\%)$ & $70.4 \pm 7.4[57-80]$ \\
\hline Education, years & $7.6 \pm 3.9[3-18]$ & $6.6 \pm 3.6[4-18]$ & $24(\%)$ \\
\hline Mini mental state exam & $27.2 \pm 1.7[23-30]$ & $26.9 \pm 1.3[23-29]$ & $7.6 \pm 3.7[3-17]$ \\
\hline Alpha3/alpha2 & $1.09 \pm 0.15[0.77-1.52]$ & $1.29 \pm 0.1^{*}+[1.17-1.52]$ & $27 . \pm 1.7[24-30]$ \\
\hline
\end{tabular}

Table 1: Demographic and cognitive characteristics in the whole sample, disaggregated for increased levels of Alpha3/ Alpha2 ratio. Numbers denote mean \pm standard deviation, number and [range]. $p$ denotes significance on ANOVA (continuous variables) and chi-square test (dichotomous variables). 
that band. The alpha3/alpha2 was computed in all subjects and three groups were obtained according to increasing tertiles values of alpha3/ alpha2: low $(\mathrm{a} 3 / \mathrm{a} 2<1)$ middle $(1<\mathrm{a} 3 / \mathrm{a} 2<1.16)$ and high $(\mathrm{a} 3 / \mathrm{a} 2>1.17)$. The three groups of MCI have been demostrated in previous studies to be different in nature. In particular, the high alpha3/alpha 2 EEG power ratio MCI group is at major risk to convert to Alzheimer's disease [29], as well as to have different pattern of hippocampal atrophy [28] and basal ganglia and thalamus gray matter lesions $[29,15]$ as compared to the other alpha3/alpha2 power ratio MCI groups. Moreover, this group subdivision has been chosen for reason of homogeneity and comparability with the previous studies

\section{MRI scans}

For each subject, a high-resolution sagittal $\mathrm{T} 1$ weighted volumetric MR scan was acquired by using a 1.0 T Philips Gyroscan scanner, with a gradient echo $3 \mathrm{D}$ technique: $\mathrm{TR}=20 \mathrm{~ms}, \mathrm{TE}=5 \mathrm{~ms}$, flip angle $=30$, field of view $=220 \mathrm{~mm}$, acquisition matrix $256 \times 256$, slice thickness $1.3 \mathrm{~mm}$. The pattern of gray matter atrophy was studied using the Voxel Based Morphometry technique [32].

\section{Voxel-based morphometry}

3D images were processed through SPM5 software package (Statistical Parametric Mapping, Version 5; Wellcome Department of Imaging Neuroscience, London, UK; http://www.fil.ion.ucl.ac.uk/ spm), running on Matlab 7.0.1 (Math-Works, Natick, MA, USA). DICOM files were converted in ANALYZE format image, the extracranial voxels were removed and the anterior commissure (AC) was manually set for all images as the origin of the spatial coordinates for an anatomical normalization algorithm implemented in SPM. Converted files were then segmented into gray and white matter and normalized to the GM population templates, generated from the complete image set, using the Diffeomorphic Anatomical Registration using Exponentiated Lie algebra (DARTEL) registration method [32].This non-linear warping technique minimizes between-subject structural variations.

Spatially normalized images were modulated by the Jacobian determinants derived from the spatial normalization, to ensure that the overall amount of each tissue class was not altered by the spatial normalization procedure. The final voxel resolution after DARTEL was $1.5 \times 1.5 \times 1.5 \mathrm{~mm}$. Finally each modulated, warped GM image was transformed to MNI space and smoothed with an $8 \mathrm{~mm}$ full-width at half-maximum (FWHM) Gaussian kernel filter. The experimenter performing the MRN computations was blinded to the results of previous EEG works, so that there were not biases in the analysis.

\section{Statistical analysis}

VBM results were assessed at an uncorrected threshold of $\mathrm{p} \leq$ 0.001. This threshold has an important limit in that it allows the type I statistical error. Anyway, a more permissive threshold could be more adequate to this explorative study, it in order to avoid the beta (or type II) statistic error, with the risk to neglect interesting results. Anyway, the power of the study was allowed by size of the sample, and by the robust results of the subsequent analyses. The sample was disaggregated into three groups according to three increasing values of alpha3/alpha2 ratio: low-a3/a2 (a3/a2<1), middle-a3/a2 $(1<\mathrm{a} 3 / \mathrm{a} 2<1.16)$, high-a3/a2 (a3/a2>1.17).

Voxel-based analyses were carried out comparing the three patient groups with increasing values of the alpha3/alpha2 ratio (high-a3/a2; middle-a3/a2; low-a3/a2).

Between-group regional differences in GM volumes were assessed by using an ANCOVA model, modelling the effects of groups (high, middle, low), and parametric nuisance covariates (age, gender, education, MMSE scores as covariates). Moreover, the total intracranial volume was introduced in the statistical analysis as a covariate, to avoid the confounding item of the global cortical atrophy. The total intracranial volume (TIV) was computed by manually tracing the entire intracranial cavity on $7 \mathrm{~mm}$ thick coronal slices, by the use of the software DISPLAY 1.3 tools. Correlation or regression analyses were not performed. The reason is that the EEG markers represents different patients population (MCI who will convert and MCI who will not), as previously demonstrated [3]. As a consequence, it is not correct to use the regression analysis because significant results should have masked. It would be possible only within each of the three groups individuated by the tertile subdivisions, but the size of each group dose not allows a powerful regression analysis.

All the analyses were restricted to the NAc as regions of interest in order to focus the relationship between there brain areas and EEG markers. It should be possible to perform a computation encompassing other brain areas, but this was beyond the scope of the present work. Moreover, the relationship of EEG markers with hippocampus and amygdala was faced in previous papers [28]. To this purpose, a mask including Caudate Nucleus, Putamen, Globus Pallidus, and NAc was entered into the models as explicit mask. It was manually traced, through the software MRIcroN, on the previous template generated from the complete image set. The detection of the anatomical regions was based on the localization of the thalamic nuclei and basal ganglia in histological sections from a human atlas [33].

\section{Results}

Table 1 shows the sociodemographic characteristics of $\mathrm{MCI}$ subgroups. When groups were defined by the tertile values of alpha3/ alpha2 the ANOVA analysis showed that there was not statistically significant differences between groups expect for alpha3/alpha2 ratio levels where all Games-Howell post hoc comparisons were significant $(\mathrm{p}<0.001)$ with progressive increasing values between groups.

\section{EEG alpha3/alpha2 ratio}

Low-a3/a2 group: No regions of GM tissue loss were found when patients with low a3/a2 ratio were compared to those with Middle and High a3/a2 ratio.

Middle-a3/a2 group: Subjects with middle a3/a2 ratio, contrasted to individuals with high a3/a2 ratios, showed more atrophy in the bilateral Accumbens nuclei, though the atrophic area was minimal in the left hemisphere (Figure 1).

No regions of significant GM tissue loss were found in other comparisons in this group.

\section{Discussion}

\section{Association between EEG markers and GM changes}

Results show that the increase of alpha3/alpha2 ratio is mostly associated with minor atrophy NAc. These results show some difference with previous studies on $\mathrm{AD}$ patients, showing basal ganglia involvement ([34-37]) in that these reported a greater atrophy of basal ganglia. Some possible explanations are: 1) in our study the evaluation of atrophy is EEG-driven, focusing on specific morphostructural features of patients; this aspect could have magnified only some GM patterns; 2) the patterns of atrophy observed in previous studies could be linked to a specific regressive processes due to aging ([38-41]) our 


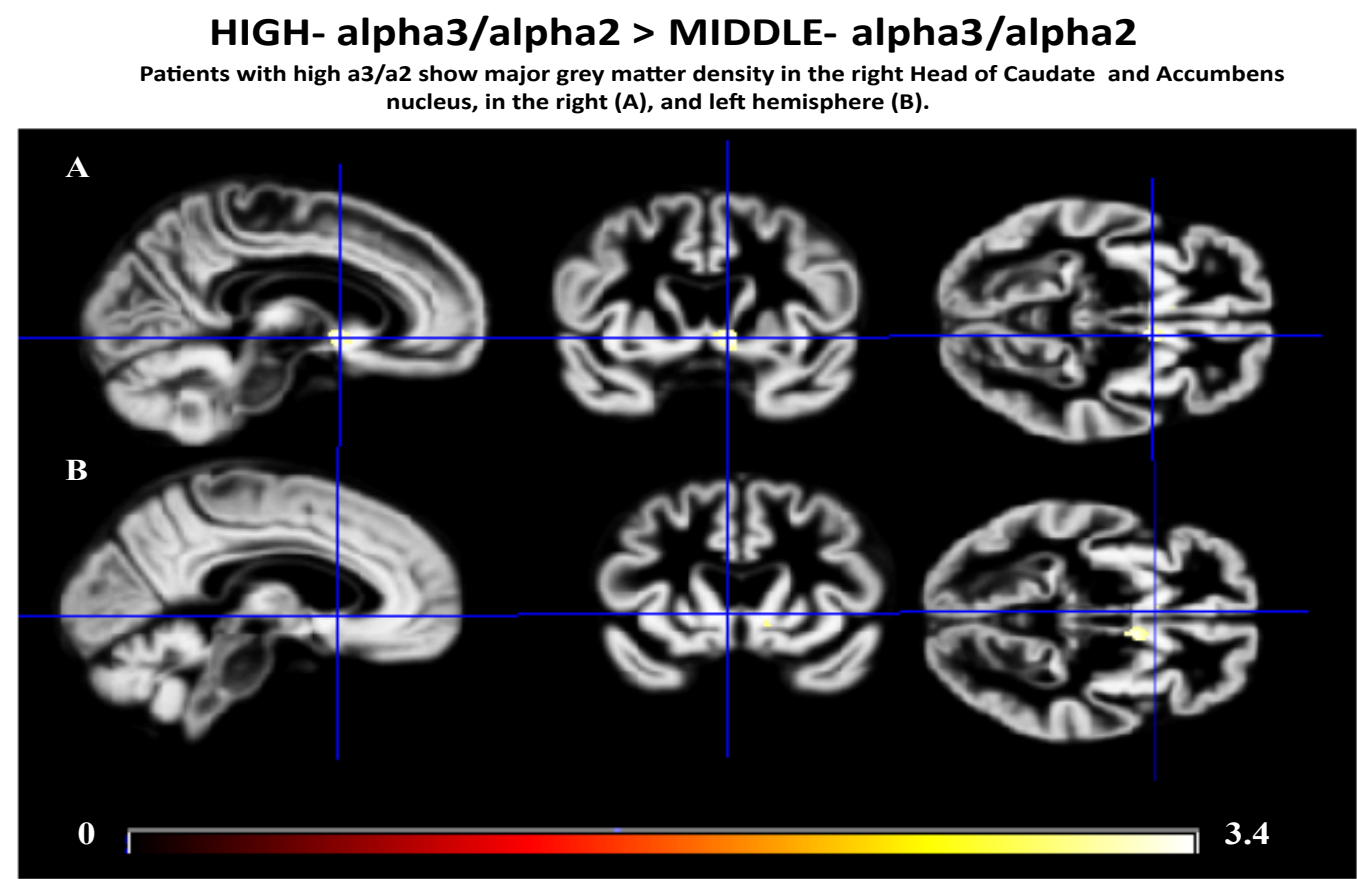

Cluster size represents the number of contiguous significant voxels. Stereotaxix coordinates reveal the position of the most significant voxel of the cluster (named "peak") in MNI space, and side denotes its anatomical localization on left $(L)$ or right $(R)$ hemisphere. Colour bar denotes T-values.

\begin{tabular}{|c|c|c|c|c|c|c|}
\hline \multicolumn{7}{|c|}{ HIGH $(a 3 / a 2>1.17)>\operatorname{MIDDLE}(1<a 3 / a 2<1.16)[\mathrm{n}=18, \mathrm{n}=38]$} \\
\hline & $\begin{array}{l}\text { Cluster size } \\
\text { (voxel number) }\end{array}$ & Side & Region & $\begin{array}{l}\text { Stereotaxic } \\
\text { coordinates }\end{array}$ & $\begin{array}{c}Z \\
\text { score }\end{array}$ & $\boldsymbol{p}$ \\
\hline $\boldsymbol{A}$ & 123 & $R$ & Accumbens & $95-3$ & 3.6 & .000 \\
\hline B & 4 & $L$ & Accumbens & $-512-2$ & 3.2 & .001 \\
\hline
\end{tabular}

Figure 1: T-map showing greater regional grey matter density in patients with high a3/a2 ratio contrasted to patients with middle a3/a2 ratio ( $p \leq .001$ uncorrected, the symbol '>' denotes 'major grey matter density then', see also table).

results are about subjects with MCI whereas previous studies were most performed on $\mathrm{AD}$ patients; it should be noted that different, perhaps compensatory, mechanisms have been suggested for MCI subjects as compared to AD patients ( [42-44]). We have not found any significant difference in NAc VBM analysis between the highest and lowest as well as the middle and the lowest alpha3/ alpha 2 ratio MCI groups. This result is somewhat cotradictory but it could be explained considering the different nature of the MCI group with highest alpha3/alpha2 ratio. Of note, this group is at major risk to develop $\mathrm{AD}$, so it is a $\mathrm{MCI}$ due to $\mathrm{AD}$ group. On the contrary, the lowest alpha3/alpha2 ratio group probably is not a neurodegenerative MCI

Anatomo-physiological relationship between EEG markers and GM changes

Our results show the presence of a specific pattern of GM changes associated with an EEG marker. The novelty emerging from our results is represented by minor atrophy of NAc in MCI patients who will develop $\mathrm{AD}$. The relatively preserved anatomical structure could suggest a state of compensatory hyperfunction of this circuitry, determining both cognitive and psychiatric symptoms of prodromal AD [45]. Indeed, the NAc is associated with the regulation of emotional, impulsivity and fear behaviour control function [8]. We argue that the increase of alpha3/alpha2 ratio is the brain electrical activity marker of these structural changes. The prevalence of an anterior circuit impinging on anterior cingulate cortex, ventral (limbic) striatum and orbito-frontal cortex, relatively spared in pre-AD patients, could be hypothesized as the anatomo-physiological network underpinning the increase of alpha 3 frequency power. On the other hand, the decrease of low alpha (alpha 2 in our analysis) rhythm could be based on the well-known disrupture of the posterior circuit, encompassing hyppocampal cortex, posterior cingulate, precuneus, posterior parietal cortices, in MCI subjects [2129].

\section{Clinical and behavioural implications}

Our results show a clear association between the increase of alpha3/alpha2 power ratio and bigger volume inside the NAc in subjects with MCI, suggesting that this network could be hyperactive in patients at major risk to develop $\mathrm{AD}$. These findings confirm a large 
body of previous literature. Recent factor analyses conducted on the Neuropsychiatric Inventory [46] collected from a large sample of AD patients have all identified factors called "behavioural dyscontrol" [47], "hyperactivity" [48], or "psychomotor syndrome" [45] that globally encompass the same symptoms (e.g., agitation, irritability, aggressivity, disinhibition, euphoria, appetite disturbances, aberrant motor behaviors). These symptoms are frequently described even in the early phase of the disease and explain a substantial part of variance of the total behavioral and psychological symptoms of dementia.

The NAc is a key entry point structure for afferent information from the periphery as well as for afferents and efferents of wider corticostriatal-pallido-thalamo-cortical functionally segregated loops (CSPTC; [49-51]). The NAc is primarily characterized by its strong inputs from limbic structures such as the amygdala, hippocampus, midline thalamus and certain regions of the prefrontal cortex, as well as from the mesolimbic dopamine system originating in the ventral tegmental area (VTA). Impulsive acts and decisions are related to individual differences in the neural representations of stimuli/events [52]. The NAc plays an important regulatory role in the neural representation of response options, as shown by functional neuroimaging studies in healthy individuals [4]. A voluminous literature on decision-making in humans provides imaging evidence of the involvement of a variety of brain structures in impulsive choice; different subregions of the human prefrontal cortex, cingulate cortex, insula, and the amygdala are the most prominent ones [53-55]. These structures are known to either have direct anatomical connections to the NAc, or are indirectly connected [4]. The selection of immediate reinforcement has been repeatedly reported to be associated with increased activity in the NAC [9]. Imaging studies with various task have implied an association between increased NAc activity and selection of less safe option ([14,56,57]) [12]. Another possible explanation of increase in NAc and ventral striatum hyperactivity could be related to exposure associated to novel environments. A recent study has convincingly demonstrated that a modifications in glutamatergic transmission with an increase of glutamatergic receptorial subtypes occurrs in NAc after novelty exposure [58]. A modification in the shape of NAc has been demonstrated also in patients with $\mathrm{AD}$ showing sensation-seeking behavior [59]. In this optic the alpha3/alpha2 power ratio could a physiological network marker, related to a specific behavioral disorder, useful to screen out MCI subjects that could develope AD. The results of this paper have to be integrated by studies with a neuropsychiartric assessment and behavioral data to correlate with GM volumetric changes.

\section{Conclusion}

The a3/a2 ratio was associated with increase of grey matter density inside the NAc in MCI subjects at major risk to develop AD. Further studies will investigate the salience of this result in early diagnosis and the association of this EEG marker with cognitive impairment in prodromal $\mathrm{AD}$ patients.

\section{Acknowledgements}

The work is funded by Fatebenefratelli Association for Research.

\section{References}

1. Stam CJ, van der Made Y, Pijnenburg YA, Scheltens P (2003) EEG synchronization in mild cognitive impairment and Alzheimer's disease. Acta Neurol Scand 108: 90-96.

2. Rossini PM, Buscema M, Capriotti M, Grossi E, Rodriguez G, et al. (2008) Is it possible to automatically distinguish resting EEG data of normal elderly vs. mild cognitive impairment subjects with high degree of accuracy? Clin Neurophysiol 119: 1534-1545.
3. Moretti DV, Pievani M, Geroldi C, Binetti G, Zanetti O, et al. (2009) Increasing hippocampal atrophy and cerebrovascular damage is differently associated with functional cortical coupling in $\mathrm{MCl}$ patients. Alzheimer Dis Assoc Disord 23: 323-332.

4. Rochat L, Delbeuck X, Billieux J, d'Acremont $M$, Van der Linden AC, et al. (2008) Assessing impulsivity changes in Alzheimer disease. Alzheimer Dis Assoc Disord 22: 278-283.

5. Folstein MF, Folstein SE, McHugh PR, (1975) 'Mini mental state': a practica method for grading the cognitive state of patients for clinician. J Psychiatr Res 12: 189-198.

6. Mattis M (1976) Mental status examination for organic mental syndrome in the elderly patient. In Bellack L (Ed), Geriatric Psychiatry: A Handbook for Psychiatrists and Primary Care Physicians. New York: Grune and Stratton 77121

7. Rochat L, Billieux J, Juillerat Van der Linden AC, Annoni JM, Zekry D, et al. (2013) A multidimensional approach to impulsivity changes in mild Alzheimer's disease and control participants: cognitive correlates. Cortex 49: 90-100.

8. Basar K, Sesia T, Groenewegen H, Steinbusch HW, Visser-Vandewalle V, et al. (2010) Nucleus accumbens and impulsivity. Prog Neurobiol 92: 533-557.

9. McClure SM, Laibson DI, Loewenstein G, Cohen JD (2004). Separate neura systems value immediate and delayed monetary rewards. Science 306: 503 507.

10. McClure SM, Ericson KM, Laibson DI, Loewenstein G, Cohen JD (2007) Time discounting for primary rewards. J Neurosci 27: 5796-5804

11. Boettiger CA, Mitchell JM, Tavares VC, Robertson M, Joslyn G, et al. (2007) Immediate reward bias in humans: fronto-parietal networks and a role for the catechol-O-methyltransferase 158(Val/Val) genotype. J Neurosci 27: 1438314391.

12. Weber BJ, Huettel SA (2008) The neural substrates of probabilistic and intertemporal decision making. Brain Res 1234: 104-115.

13. Wittmann M, Leland DS, Churan J, Paulus MP (2007) Impaired time perception and motor timing in stimulant-dependent subjects. Drug Alcohol Depend 90: 183-192.

14. Kuhnen CM, Knutson B (2005) The neural basis of financial risk taking. Neuron 47: 763-770.

15. Moretti DV, Paternicò D, Binetti G, Zanetti O, Frisoni GB (2012) EEG markers are associated to gray matter changes in thalamus and basal ganglia in subjects with mild cognitive impairment. Neuroimage 60: 489-496.

16. Hughes CP, Berg L, Danziger WL, Coben LA, Martin RL (1982) A new clinical scale for the staging of dementia. Br J Psychiatry 140: 566-572.

17. Rosen WG, Terry RD, Fuld PA, Katzman R, Peck A (1980) Pathological verification of ischemic score in differentiation of dementias. Ann Neurol 7: 486488 .

18. Lawton MP, Brody EM (1969) Assessment of older people: self-maintaining and instrumental activities of daily living. Gerontologist 9: 179-186.

19. Lezak M, Howieson D, Loring DW (2004) Neuropsychological Assessment, fourth edition. Oxford: University Press.

20. Radloff LS (1977) The CES-D scale: A self-report depression scale for research in the general population. Applied Psychological Measurement 1: 385-401

21. Moretti DV, Babiloni F, Carducci F, Cincotti F, Remondini E, et al. (2003) Computerized processing of EEG-EOG-EMG artifacts for multi-centric studies in EEG oscillations and event-related potentials. Int J Psychophysiol 47: 199216.

22. Moretti DV, Babiloni C, Binetti G, Cassetta E, Dal Forno G, et al. (2004) Individual analysis of EEG frequency and band power in mild Alzheimer's disease. Clin Neurophysiol 115: 299-308.

23. Moretti DV, Miniussi C, Frisoni G, Zanetti O, Binetti G et al. (2007) Vascular damage and EEG markers in subjects with mild cognitive impairment. Clinical neurophysiology 118: 1866-1876.

24. Moretti DV, Miniussi C, Frisoni GB, Geroldi C, Zanetti O et al. (2007) Hippocampal atrophy and EEG markers in subjects with mild cognitive impairment. Clinical neurophysiology 118: 2716-2729.

25. Moretti DV, Frisoni GB, Pievani M, Rosini S, Geroldi C, et al. (2008) 
Cerebrovascular disease and hippocampal atrophy are differently linked to functional coupling of brain areas: an EEG coherence study in $\mathrm{MCl}$ subjects. J Alzheimers Dis 14: 285-299.

26. Moretti DV, Pievani M, Fracassi C, Geroldi C, Calabria M, et al. (2008) Brain vascular damage of cholinergic pathways and EEG markers in mild cognitive impairment. J Alzheimers Dis 15: 357-372.

27. Moretti DV, Fracassi C, Pievani M, Geroldi C, Binetti G, et al. (2009) Increase of theta/gamma ratio is associated with memory impairment. Clin Neurophysiol 120: 295-303.

28. Moretti DV, Pievani M, Fracassi C, Binetti G, Rosini S, et al. (2009) Increase of theta/gamma and alpha3/alpha2 ratio is associated with amygdalohippocampal complex atrophy. J Alzheimers Dis 17: 349-357.

29. Moretti DV, Frisoni GB, Fracassi C, Pievani M, Geroldi C, et al. (2011) MC patients' EEGs show group differences between those who progress and those who do not progress to AD. Neurobiol Aging 32: 563-571.

30. Klimesch W (1997) EEG-alpha rhythms and memory processes. Int J Psychophysiol 26: 319-340.

31. Klimesch W (1999) EEG alpha and theta oscillations reflect cognitive and memory performance: a review and analysis. Brain Res Brain Res Rev 29 169-195.

32. Ashburner J (2007) A fast diffeomorphic image registration algorithm Neuroimage 38: 95-113.

33. Mai JK, Assheuer J, Paxinos G (1997) Atlas of the Human Brain. Academic Press, San Diego

34. de Jong LW, van der Hiele K, Veer IM, Houwing JJ, Westendorp RG, et al. (2008) Strongly reduced volumes of putamen and thalamus in Alzheimer's disease: an MRI study. Brain 131: 3277-3285.

35. Canu E, McLaren DG, Fitzgerald ME, Bendlin BB, Zoccatelli G et al. (2010) Microstructural diffusion changes are independent of macrostructural volume loss in moderate to severe Alzheimer's disease. J Alzheimers Dis 19: 963-976.

36. Cherubini A, Péran P, Spoletini I, Di Paola M, Di lulio F, et al. (2010) Combined volumetry and DTI in subcortical structures of mild cognitive impairment and Alzheimer's disease patients. J Alzheimers Dis 19: 1273-1282.

37. Zarei M, Patenaude B, Damoiseaux J, Morgese C, Smith S, et al. (2010) Combining shape and connectivity analysis: an MRI study of thalamic degeneration in Alzheimer's disease. Neuroimage 49: 1-8.

38. Cabeza R, Daselaar SM, Dolcos F, Prince SE, Budde M, et al. (2004) Taskindependent and task-specific age effects on brain activity during working memory, visual attention and episodic retrieval. Cereb Cortex 14: 364-375.

39. Logan JM, Sanders AL, Snyder AZ, Morris JC, Buckner RL (2002) Underrecruitment and nonselective recruitment: dissociable neural mechanisms associated with aging. Neuron 33: 827-840.

40. Park DC, Welsh RC, Marshuetz C, Gutchess AH, Mikels J, et al. (2003) Working memory for complex scenes: age differences in frontal and hippocampa activations. J Cogn Neurosci 15: 1122-1134.

41. Grady CL, Springer MV, Hongwanishkul D, Mclntosh AR, Winocur G (2006) Age-related changes in brain activity across the adult lifespan. J Cogn Neurosci 18: $227-241$
42. Dickerson BC, Salat DH, Bates JF, Atiya M, Killiany RJ, et al. (2004) Media temporal lobe function and structure in mild cognitive impairment. Ann Neuro 56: 27-35.

43. Dickerson BC, Salat DH, Greve DN, Chua EF, Rand-Giovannetti E, et al. (2005) Increased hippocampal activation in mild cognitive impairment compared to normal aging and AD. Neurology 65: 404-411.

44. Golob EJ, Irimajiri R, Starr A (2007) Auditory cortical activity in amnestic mild cognitive impairment: relationship to subtype and conversion to dementia. Brain 130: 740-752.

45. Spalletta G, Musicco M, Padovani A, Rozzini L, Perri R, et al. (2010) Neuropsychiatric symptoms and syndromes in a large cohort of newly diagnosed, untreated patients with Alzheimer disease. Am J Geriatr Psychiatry 18: $1026-1035$

46. Cummings JL, Mega M, Gray K, Rosenberg-Thompson S, Carusi DA, et al. (1994) The Neuropsychiatric Inventory: comprehensive assessment of psychopathology in dementia. Neurology $44: 2308-2314$

47. Hollingworth P, Hamshere ML, Moskvina V, Dowzell K, Moore PJ, et al. (2006) Four components describe behavioral symptoms in 1,120 individuals with lateonset Alzheimer's disease. J Am Geriatr Soc 54: 1348-1354.

48. Aalten P, Verhey FR, Boziki M, Bullock R, Byrne EJ, et al. (2007) Neuropsychiatric syndromes in dementia. Results from the European Alzheime Disease Consortium: part I. Dement Geriatr Cogn Disord 24: 457-463.

49. Steriade M (2006) Grouping of brain rhythms in corticothalamic systems Neuroscience 137: 1087-1106.

50. Kopell BH, Greenberg BD (2008) Anatomy and physiology of the basal ganglia: implications for DBS in psychiatry. Neurosci Biobehav Rev 32: 408-422.

51. Haber SN, Calzavara R (2009) The cortico-basal ganglia integrative network the role of the thalamus. Brain Res Bull 78: 69-74.

52. Chambers RA, Potenza MN (2003) Neurodevelopment, impulsivity, and adolescent gambling. J Gambl Stud 19: 53-84

53. Ernst M, Paulus MP (2005) Neurobiology of decision making: a selective review from a neurocognitive and clinical perspective. Biol Psychiatry 58: 597-604.

54. Knutson B, Bossaerts $P$ (2007) Neural antecedents of financial decisions. $J$ Neurosci 27: 8174-8177.

55. Rushworth MF, Behrens TE (2008) Choice, uncertainty and value in prefronta and cingulate cortex. Nat Neurosci 11: 389-397.

56. Ernst M, Nelson EE, McClure EB, Monk CS, Munson S (2004) Choice selection and reward anticipation: an fMRI study. Neuropsychologia 42: 1585-1597.

57. Matthews SC, Simmons AN, Lane SD, Paulus MP (2004) Selective activation of the nucleus accumbens during risk-taking decision making. Neuroreport 15 2123-2127.

58. Rothwell PE, Kourrich S, Thomas MJ (2011) Environmental novelty cause stress-like adaptations at nucleus accumbens synapses: implications for studying addiction-related plasticity. Neuropharmacology 61: 1152-1159.

59. de Jong LW, Ferrarini L, van der Grond J, Milles JR, Reiber JH, et al. (2011) Shape abnormalities of the striatum in Alzheimer's disease. J Alzheimers Dis 23: $49-59$. 